\title{
Elastic Properties and Stacking Fault Energies of Borides, Carbides and Nitrides from First-Principles Calculations
}

\author{
Yong Zhang ${ }^{1} \cdot$ Zi-Ran Liu $^{2} \cdot$ Ding-Wang Yuan ${ }^{1} \cdot$ Qin Shao ${ }^{1}$ Jiang-Hua Chen ${ }^{1} \cdot$ Cui-Lan Wu ${ }^{1} \cdot$ Zao-Li Zhang $^{3}$
}

Received: 7 September 2018 / Revised: 31 October 2018 / Published online: 4 February 2019

(c) The Chinese Society for Metals (CSM) and Springer-Verlag GmbH Germany, part of Springer Nature 2019

\begin{abstract}
Owing to the excellent elastic properties and chemical stability, binary metal or light element borides, carbides and nitrides have been extensively applied as hard and low-compressible materials. Researchers are searching for harder materials all the time. Recently, the successful fabrication of nano-twinned cubic BN (Tian et al. Nature 493:385-388, 2013) and diamond (Huang et al. Nature 510:250-253, 2014) exhibiting superior properties than their twin-free counterparts allows an efficient way to be harder. From this point of view, the borides, carbides and nitrides may be stronger by introducing twins, whose formation tendency can be measured using stacking fault energies (SFEs). The lower the SFEs, the easier the formation of twins. In the present study, by means of first-principles calculations, we first calculated the fundamental elastic constants of forty-two borides, seventeen carbides and thirty-one nitrides, and their moduli, elastic anisotropy factors and bonding characters were accordingly derived. Then, the SFEs of the $\{111\}<112>$ glide system of twenty-seven compounds with the space group $\mathrm{F} \overline{4} 3 \mathrm{~m}$ or $\mathrm{Fm} \overline{3} \mathrm{~m}$ were calculated. Based on the obtained elastic properties and SFEs, we find that (1) light element compounds usually exhibit superior elastic properties over the metal borides, carbides or nitrides; (2) the $5 d$ transitionmetal compounds $\left(\mathrm{ReB}_{2}, \mathrm{WB}, \mathrm{OsC}, \mathrm{RuC}, \mathrm{WC}, \mathrm{OsN}_{2}, \mathrm{TaN}\right.$ and $\left.\mathrm{WN}\right)$ possess comparable bulk modulus $(B)$ with that of cBN $(B=363 \mathrm{GPa})$; (3) twins may form in $\mathrm{ZrB}, \mathrm{HfN}, \mathrm{PtN}, \mathrm{VN}$ and $\mathrm{ZrN}$, since their SFEs are lower or slightly higher than that of diamond $\left(\mathrm{SFE}=277 \mathrm{~mJ} / \mathrm{m}^{2}\right.$ ). Our work can be used as a valuable database to compare these compounds.
\end{abstract}

Keywords Inorganic compounds $\cdot$ Elastic properties $\cdot$ Stacking fault energies $\cdot$ First-principles calculations

\section{Introduction}

Due to the combination of electron-rich transition-metal (TM) atoms and strong covalent-bonded atoms (e.g., B, C and $\mathrm{N}$ ), most of the binary TM borides, carbides and nitrides are mechanically hard and incompressible materials [1-3]. They show relative high modulus, ultra-high melting point

Available online at http://link.springer.com/journal/40195

Jiang-Hua Chen

jhchen123@hnu.edu.cn

1 Center for High-Resolution Electron Microscopy, College of Materials Science and Engineering, Hunan University, Changsha 410082, China

2 Department of Physics, Key Laboratory for Low-Dimensional Structures and Quantum Manipulation (Ministry of Education), Hunan Normal University, Changsha 410086, China

3 Erich Schmid Institute of Materials Science, Austrian Academy of Sciences, Jahnstraße 12, 8700 Leoben, Austria and good chemical stability. Hence, they are widely used or have potential in cutting, wear- and corrosion-resistant coating fields [1, 4-6]. For instance, chromium nitride and titanium nitride coatings can enhance the performance of cutting tools $[5,7]$. As reinforcements, TiB whiskers or $\mathrm{SiC}$ fibers could be added into alloys or ceramics to fabricate composite materials [8-11]. They also might serve as functional materials. For example, magnesium diboride has been proved to be a superconductor [12]. Nickel and cobalt borides are known for their magnetic properties and could be used as catalyst for hydrogen production [13].

Researchers have never stopped searching for super-hard and ultra-incompressible materials. Many experiments and ab initio calculations have conducted, and these works mainly focus on the combination of $3 d-5 d$ TMs with light elements (B, C and $\mathrm{N}$ ) or the light elements with themselves [14-21]. The experimental synthesized $\mathrm{PtN}$ [15] and $\mathrm{PtN}_{2}$ [16] and the first-principles calculation-predicted $\mathrm{Re}_{4} \mathrm{C}$ [18] have comparable bulk moduli with $\mathrm{cBN}(\sim 379 \mathrm{GPa})$. Nano-twinned cBN [22] and diamond [23], which are 
much harder than their twin-free counterparts, have been successfully fabricated from onion-like precursors at high temperature and high pressure. Twinning can also induce elastic strengthening in SiC nano-wires [24]. Recently, the SFEs and twinnability have been theoretically studied for the group IV TM carbides [25, 26]. The twin boundaries can serve as barriers for dislocation motion or be regarded as grain boundaries, which can decrease the grain size. The Hall-Petch relationship $\sigma$ is proportional to $d^{-1 / 2}(\sigma$ denotes yield strength or hardness and $d$ is the grain size) [27, 28], showing that the smaller the grain size, the stronger (the harder) the material. Previous reported twinned cBN, diamond and $\mathrm{SiC}$ possess excellent elastic properties; therefore, twinning is helpful for improving the hardness. The tendency for twin formation can be measured by stacking fault energies (SFEs, $\gamma$ ), which is an intrinsic parameter for one material at finite temperature. More twins are found in alloys with lower SFEs [29-31]. For instance, twins are frequently detected in $\mathrm{Cu}-16$ at. $\% \mathrm{Al}\left(\gamma \sim 6 \mathrm{~mJ} / \mathrm{m}^{2}\right)$ than $\mathrm{Cu}-2.3$ at.\% $\mathrm{Al}$ $\left(\gamma \sim 48 \mathrm{~mJ} / \mathrm{m}^{2}\right)$ [30]. Meanwhile, twins are quite common in silver $\left(\gamma \sim 18 \mathrm{~mJ} / \mathrm{m}^{2}\right)$, while they are rarely seen in coarse grain aluminum $\left(\gamma \sim 130 \mathrm{~mJ} / \mathrm{m}^{2}\right)$ [32]. Therefore, SFEs are important parameters for determining the twinning ability of borides, carbides and nitrides.

The mechanical properties of the material are closely related to the crystal structure, atomic composition, bonding and defects (e.g., twins). In this study, the elastic constants of ninety binary borides, carbides and nitrides will be carried out by employing first-principles calculations. The elastic anisotropy factors and bonding characters will be discussed. Supercells of twenty-seven compounds will also be built to model the SFEs. The materials with SFEs lower than that of diamond $\left(277 \mathrm{~mJ} / \mathrm{m}^{2}\right.$ in this study) may experience twin formation at the conditions of specific precursor, high temperature, high pressure or high strain rate. The calculated results enrich the understandings of hard and low-compressible materials.

\section{Calculation Methods and Models}

The elastic properties and SFEs are calculated by employing the Vienna ab initio simulation package (VASP) [33, 34], which is based on the density functional theory (DFT). The projector augmented wave (PAW) method [35] is used to treat the interaction between ion and core electrons. The valence electrons are described using the generalized gradient approximation (GGA) with the exchange-correlation function of Perdew, Burke and Ernzerhof (PBE) [36]. Convergence tests indicate that $600 \mathrm{eV}$ is an appropriate cutoff energy for the PAW potential to obtain fine precision in the current light-elements-contained systems, especially for elastic constants calculations. Brillouin-zone gridding is performed using the Monkhorst-Pack method [37]. Regarding that the lattice constants (LCs) of the ninety materials are diverse, all of the k-point meshes are carefully tested and tuned. Previous studies show that the nonmagnetic P6/mmm-AuB ${ }_{2}$ [17], Fm $\overline{3} \mathrm{~m}-\mathrm{ReC}$ [18] and $\mathrm{Fm} \overline{3}$ $\mathrm{m}-\mathrm{CrN}$ [38] are elastically unstable; thus, spin polarization is considered for $\mathrm{Au}, \mathrm{Re}$ and $\mathrm{Cr}$ as well as for $\mathrm{Fe}, \mathrm{Co}$ and $\mathrm{Ni}$ based compounds. Using the optimized input parameters, the calculated energy change converges to smaller than $0.2 \mathrm{meV} /$ atom and the elastic constants $C_{i j}$ change converges to $<5 \mathrm{GPa}$.

After full relaxation of the cell shape and atom positions, we employ the so-called universal linear-independent coupling strains (ULICs) method proposed by Yu et al. [39] for the elastic properties calculations. For a cubic crystal, there are three independent elastic constants $\left(C_{11}\right.$, $C_{44}$ and $C_{12}$ ), while an orthogonal (lowest symmetry in the present study) crystal has six more independent elastic constants, i.e., $C_{22}, C_{33}, C_{55}, C_{66}, C_{13}$ and $C_{23}$. The rhombohedral crystal has $C_{14}, C_{15}$ and $C_{45}$, but they are extremely small and their contributions to the elastic properties can be ignored. Based on the Voigt approximation [40], five crucial elastic properties, bulk modulus $(B)$, shear modulus $(G)$, Young's modulus $(E)$, Poisson's ratio $(v)$ and anisotropy factor $(A)$ [41], can be derived from the elastic constants:

$$
\begin{aligned}
B= & \left(C_{11}+C_{22}+C_{33}\right) / 9+2\left(C_{12}+C_{13}+C_{23}\right) / 9, \\
G= & \left(C_{11}+C_{22}+C_{33}-C_{12}-C_{13}-C_{23}\right) / 15 \\
& +\left(C_{44}+C_{55}+C_{66}\right) / 5, \\
E= & \frac{9 B G}{3 B+2 G}, \\
v= & \frac{3 B-2 G}{6 B+2 G},
\end{aligned}
$$

$A=C_{66}\left(C_{11}+2 C_{12}+C_{22}\right) /\left(C_{11} C_{12}-C_{12}^{2}\right)$ for the (001) symmetry plane of an orthogonal crystal,

$A=2 C_{66} /\left(C_{11}-C_{12}\right)$ for the (001)

symmetry plane of a tetragonal crystal,

$A=C_{33} / C_{11}$ for planes containing the [001] axis of a hexagonal crystal,

$A=\left(2 C_{44}+C_{12}\right) / C_{11}$ for the $\{001\}$

symmetry plane of a cubic crystal,

where $B$ represents the ability to resist fracture, while the reciprocal of $B$ demonstrates compressibility; $G$ represents 
the ability to resist plastic deformation. $A=1$ means a completely isotropy material, while $A$ larger or smaller than unity indicates the degree of elastic anisotropy [42].

The perfectly close-packed stacking sequence of an $\mathrm{F} \overline{4} 3 \mathrm{~m}$ or $\mathrm{Fm} \overline{3} \mathrm{~m}$ crystal can be expressed as …A $\alpha \mathrm{B} \beta \mathrm{C} \gamma \mathrm{A} \alpha \mathrm{B} \beta \mathrm{C} \gamma \mathrm{A} \cdots$. The crystal structure with a space group (SG) of $\mathrm{F} \overline{4} 3 \mathrm{~m}$ has two stacking fault sequences $\cdots \mathrm{A} \alpha \mathrm{B} \beta \mathrm{C} \alpha \mathrm{B} \beta \mathrm{C} \gamma \mathrm{A} \cdots$ (denotes as SF1) and $\cdots \mathrm{A} \alpha \mathrm{B} \beta \mathrm{C} \gamma \mathrm{B} \beta \mathrm{C} \gamma \mathrm{A} \cdots(\mathrm{SF} 2)$ for the $\{111\}<112>$ glide system. Similarly, the crystal with SG of $F \bar{m} \overline{3} \mathrm{~m}$ also has SF1 $(\cdots \mathrm{A} \alpha \mathrm{B} \beta \mathrm{B} \alpha \mathrm{B} \beta \mathrm{C} \gamma \mathrm{A} \cdots)$ ) and SF2 (․ㅅ $\alpha \mathrm{B} \beta \mathrm{C} \beta \mathrm{B} \beta \mathrm{C} \gamma \mathrm{A} \cdots)$ for the $\{111\}<112>$ glide system, as shown in Fig. 1. A supercell was built with five layers and four atoms in each layer; here, $\mathrm{A} \alpha$ or $\mathrm{B} \beta$ or $\mathrm{C} \gamma$ is regarded as one layer. For calculating the SFEs, an SF lying in the middle of [111] direction is employed. To evaluate the influence between two adjacent SFs along the [111] direction, larger supercells with seven to eleven layers were tested. Tests show that five layers' supercell was large enough to eliminate the interactions with the nearest SFs. Thus, the five layers' supercell is employed for the SFEs calculations. The SFE $\gamma$ is defined as [43, 44]:

$\gamma=\left(E_{\mathrm{sf}}-E_{\mathrm{p}}\right) / S$,

where $S$ is the cross area of SF, $E_{\mathrm{sf}}$ is the energy of a supercell containing one SF, and $E_{\mathrm{p}}$ is the energy of a perfect supercell which has the same atoms as the faulted supercell.

\section{Results and Discussion}

\subsection{Elastic Properties}

The elastic properties can be estimated by nano-indentation, tensile testing, ultrasonic measurements or other experimental methods. However, the experimental values of elastic properties are sensitive to sample preparation, anisotropy and testing methods. Ab initio calculations have been widely used in the determination of elastic properties of the group IV-VI TM (Ti, V, Cr, W, etc.) borides (carbides and nitrides) and show decent agreement with experiments [3, $45,46]$. Here, the calculated lattice constants (LCs) and elastic properties are listed in Tables 1, 2 and 3 for the forty-two borides, seventeen carbides and thirty-one nitrides, respectively. Generally, many elastic-related properties, including moduli, anisotropy factors, bonding characters and Debye temperature [47], can be obtained using the elastic constants. Our calculated results are comparable with the results from the literature (see details in Tables 1, 2, 3). Slight varieties which mainly arise from the input parameters during calculation still exist. For instance, the elastic constants of unrelaxed crystal are different from that of relaxed one [48]; the local density approximation (LDA) pseudopotential usually results in a larger elastic constants than GGA [20, 42, 46]. It should be noted that, during the calculations of elastic constants, the GGA pseudopotential is chosen and the lattice parameters are fully relaxed.

The elastic stability criteria that concern independent elastic constants for a cubic crystal should satisfy $C_{11}>0$, $C_{44}>0, C_{11}>C_{12},\left(C_{11}+2 C_{12}\right)>0$; for a hexagonal one $C_{11}>C_{12}, C_{12}>0, C_{33}>0, C_{44}>0,\left(C_{11}+C_{12}\right) C_{33}-2 C_{13}^{2}>0$; for a tetragonal crystal $C_{11}>0, C_{33}>0, C_{44}>0, C_{66}>0$, $C_{11}>C_{12}, C_{11}+C_{33}>2 C_{13},\left(2 C_{11}+C_{33}+2 C_{12}+4 C_{13}\right)>0$; and for an orthorhombic $C_{11}>0, C_{22}>0, C_{33}>0, C_{44}>0$, $C_{55}>0, C_{66}>0, C_{11}+C_{22}>2 C_{12}, C_{11}+C_{33}>2 C_{13}$, $C_{22}+C_{33}>2 C_{23},\left(C_{11}+C_{22}+C_{33}+2 C_{12}+2 C_{13}+2 C_{23}\right)>0$ [42]. The elastic instability is corresponding to the energetic instability [17]. Based on the above criteria, among the ninety phases, the $\mathrm{P} 6 / \mathrm{mmm}-\mathrm{AuB}_{2}$ [17], $\mathrm{Fm} \overline{3} \mathrm{~m}-\mathrm{ReC}$ [18] and

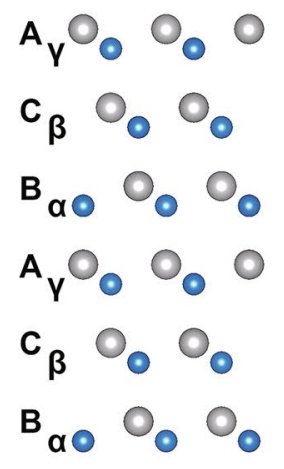

A

(a)

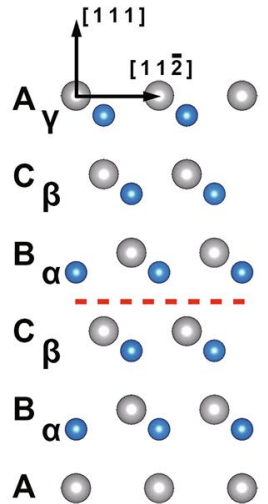

(b)

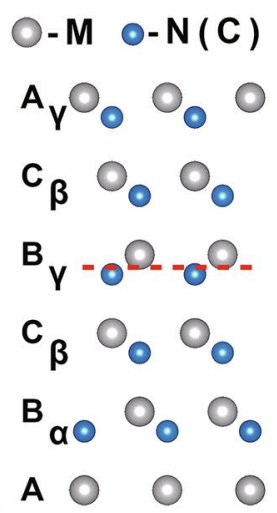

(c)

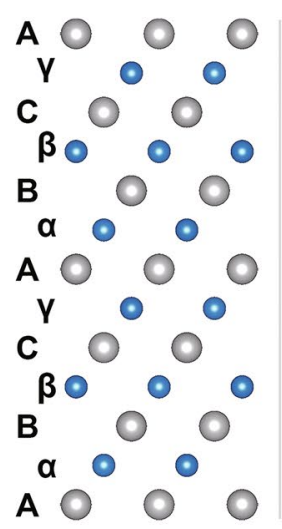

(d)

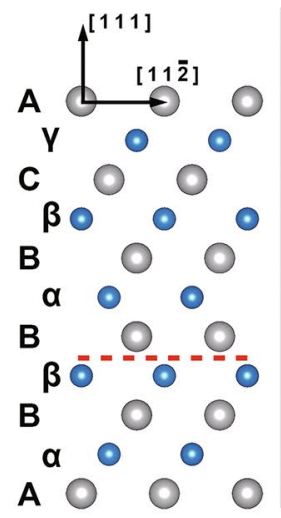

(e)

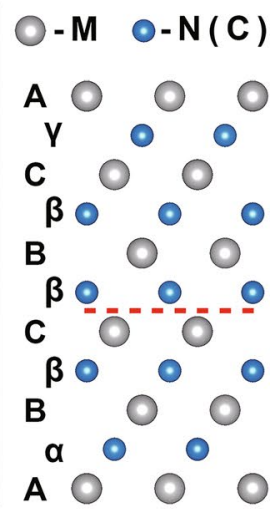

(f)
Fig. 1 Illustration of SF structure for SG F $\overline{4} 3 \mathrm{~m} \mathbf{a}, \mathbf{b}, \mathbf{c}$ and $\mathrm{Fm} \overline{3} \mathrm{~m} \mathbf{d}$, e, f. Horizontal direction [112] , vertical direction [111], all viewed from [110]. a, d Perfect supercells, b, e SF1, c, f SF2. Red dash line denotes the SF. Gray and blue spheres represent metal atom and N (or C) atoms, respectively 


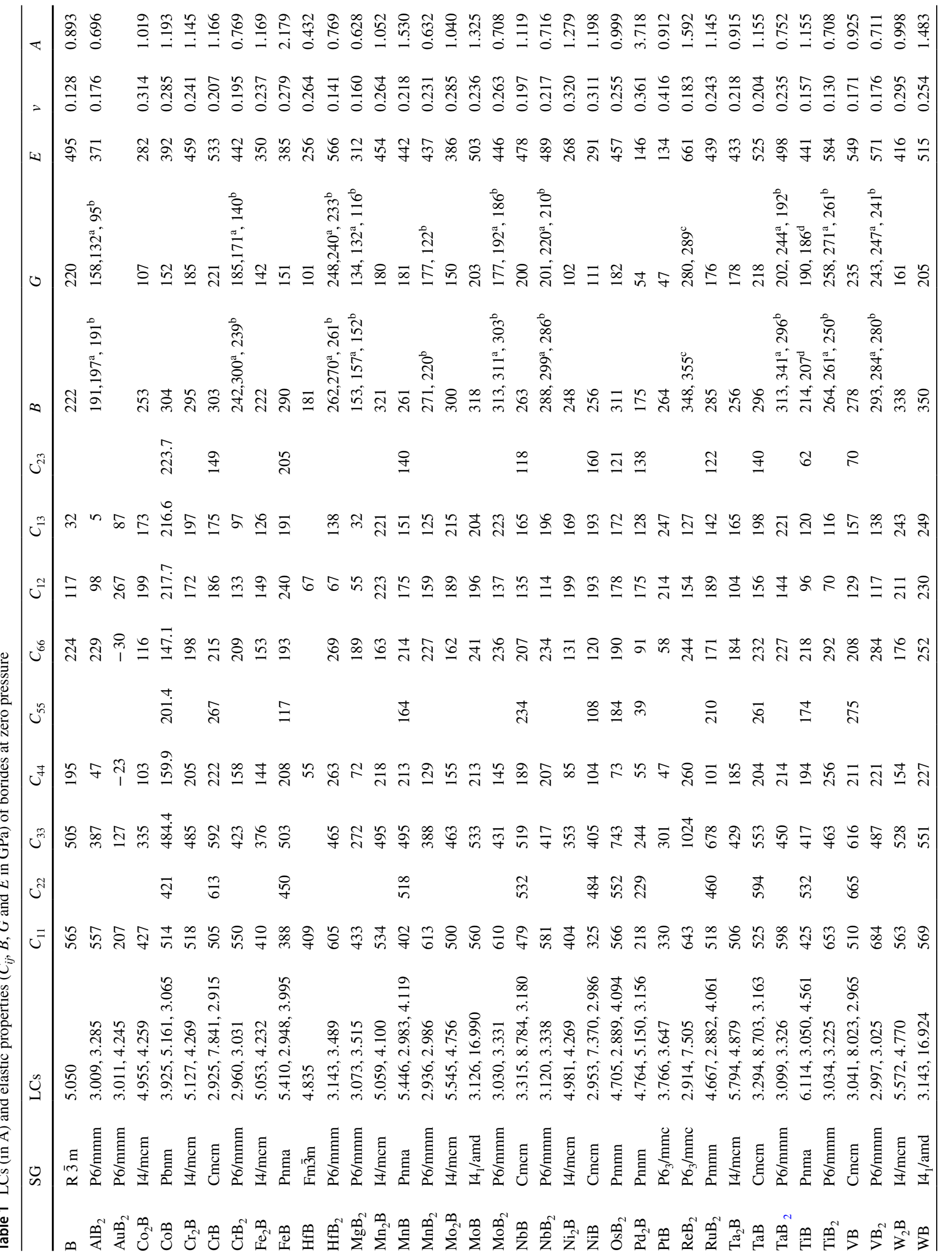




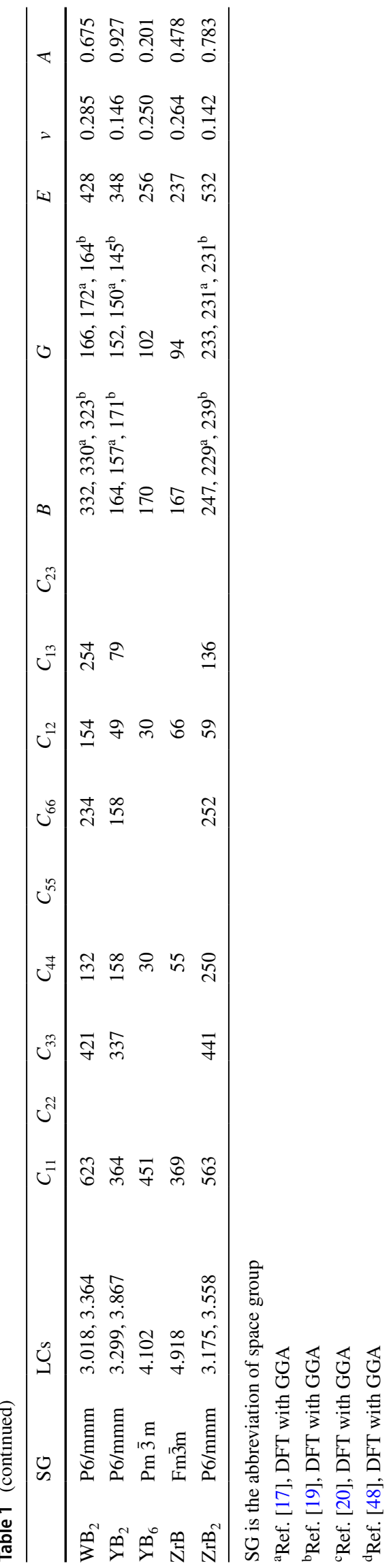

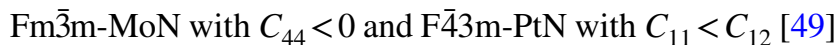
are elastically unstable. Besides, Zhou et al. [38] suggest the non-magnetic state $\mathrm{Fm} \overline{3} \mathrm{~m}$-CrN should also be elastically unstable because $C_{44}$ is quite small ( $\sim 6 \mathrm{GPa}$ ), and the spin polarization should be considered.

There are mainly three types of metal borides: $\mathrm{M}_{2} \mathrm{~B}$ (tetragonal), $\mathrm{MB}$ (orthogonal) and $\mathrm{MB}_{2}$ (hexagonal). Much theoretical attention has been paid on the $\mathrm{MB}_{2}$ type but not the former two $[17,19,50]$. Here, some interesting conclusions could be drawn by comparing the elastic properties of the three phases from the data listed in Table 1. Generally, the bulk modulus $B$ and shear modulus $E$ of $\mathrm{MB}_{2}$ are larger than that of MB for group IV and V TM, while it is opposite for group VI TM. Most of the group IV-VII TM borides all exhibit higher elastic properties than boron. At the same time, the values of $B$ for borides within one group are similar. The $5 d$ TM borides possess pronounced elastic properties among the forty-two borides. From group IV to VI, the anisotropy character decreases for the $3 d$ TM borides while increases for the $4 d$ and $5 d$ phases. But no special trends of $B, G$ and $E$ are found for $3 d, 4 d$ or $5 d$ TM borides. These regularities of the borides can be explained by the crystal structures, bonding characters and electronic configurations $[3,50]$. Among these borides, $\mathrm{ReB}_{2}$ and $\mathrm{WB}$ give the largest $B \sim 350 \mathrm{GPa}$ which is comparable with that of cBN ( $363 \mathrm{GPa}$ ). However, the experimental $B$ of $\mathrm{ReB}_{2}$ is only $173 \mathrm{GPa}$ [51], probably due to the strong anisotropy ( $A=1.59)$ or the as-fabricated $\mathrm{ReB}_{2}$ is not fully dense. All the $A$ values of seven orthogonal MB (except for $\mathrm{FeB}$ and $\mathrm{MnB}$ ) as well as eight tetragonal $\mathrm{M}_{2} \mathrm{~B}$ are slightly lower or higher than unity which indicates weak anisotropy for the (001) symmetry plane of these phases. Except for $\mathrm{ReB}_{2}$, the hexagonal borides usually exhibit favorable elastic properties and unobvious anisotropy $(A>0.63)$, while cubic ones have poor elastic properties and strong anisotropy $(A<0.48)$.

The LCs ratio $c / a$ is an important parameter for hexagonal crystals. The $B, G$ and $E$ of thirteen $\mathrm{MB}_{2}$-type (SG P6/mmm, $\mathrm{AuB}_{2}$ is excluded for elastic instability) borides against $c / a$ is plotted in Fig. 2. It shows that the three moduli decrease generally as the $c / a$ ratio increases. This trend is attributed to the stronger bonding force and shorter bond length with the smaller $c / a$ ratio. However, the electrons of one metal participating in bonding should also be considered for the scatter of the moduli. In cases, due to the lacking of bonding $d$-electrons, $\mathrm{AlB}_{2}$ and $\mathrm{MgB}_{2}$ exhibit ordinary moduli compared with TM borides; tungsten has one more $s$-electron than chromium which causes stronger bonding with $p$-electrons of boron and thus $B\left(\mathrm{CrB}_{2}\right)<B\left(\mathrm{WB}_{2}\right)$ although $c / a$ increases. What's more, besides $\mathrm{CrB}_{2}$, the difference of $B$ or $G$ is usually found less than $25 \mathrm{GPa}$ within one group (e.g., Ti, Zr, Hf) for TM diborides. 


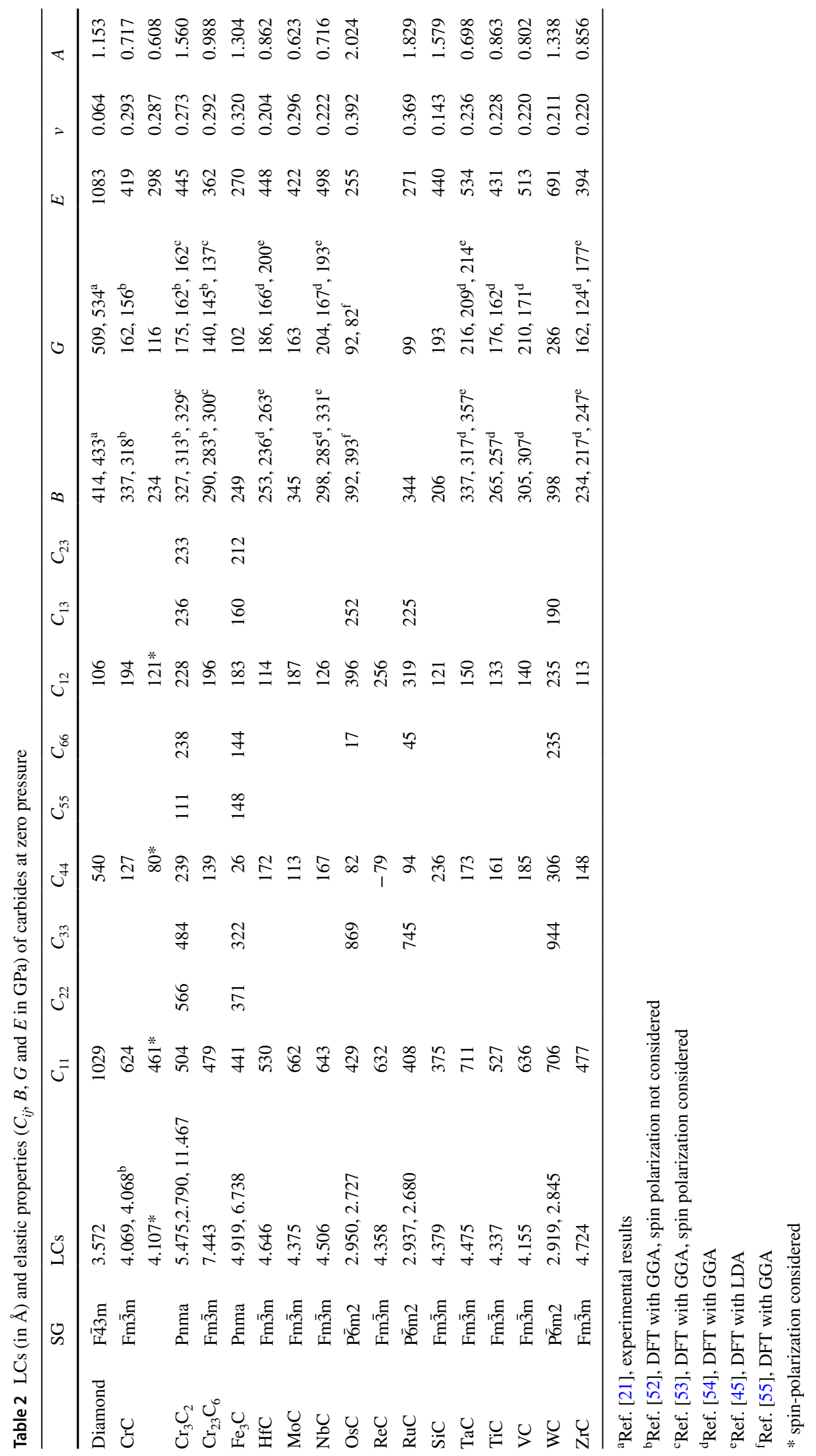




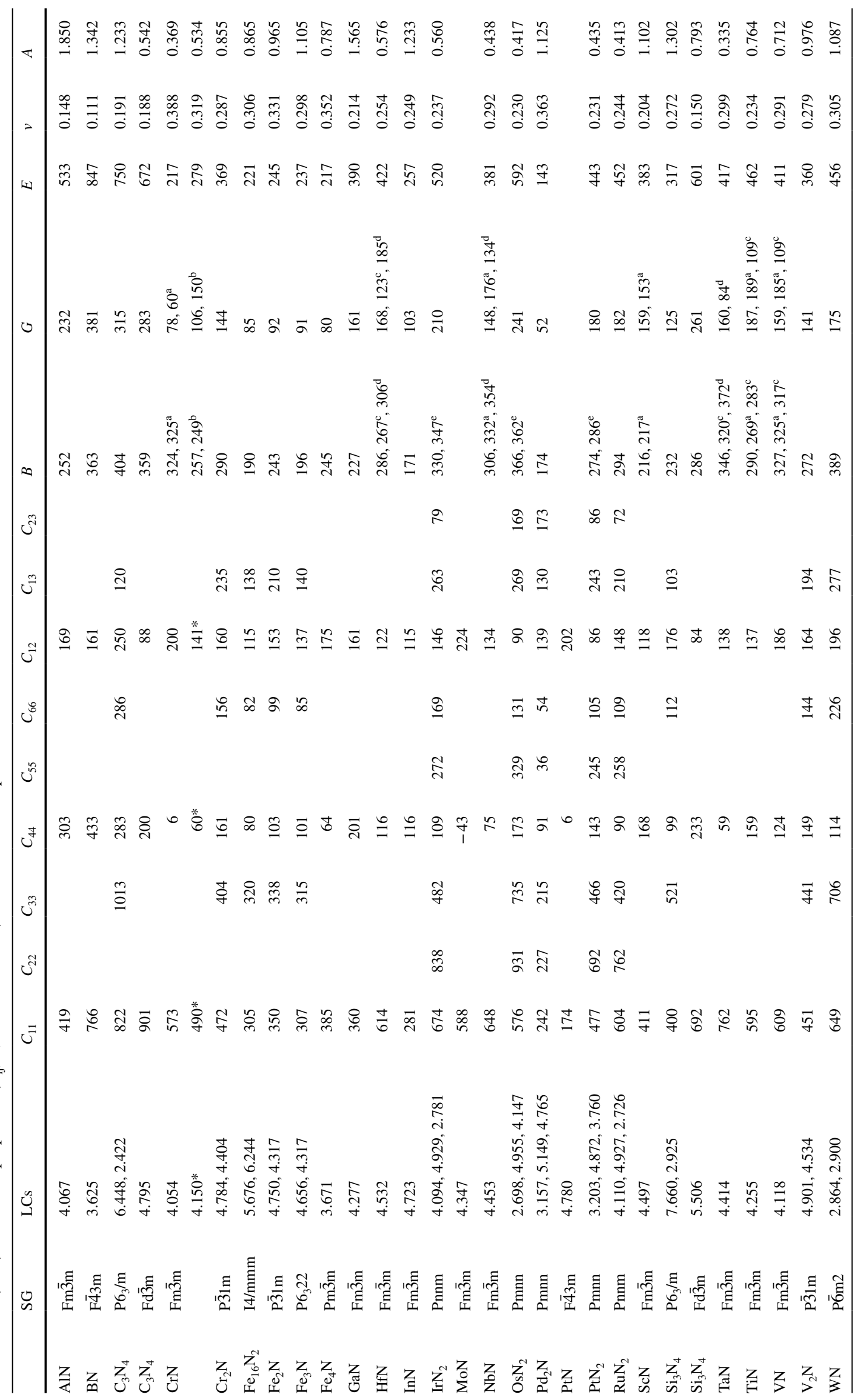



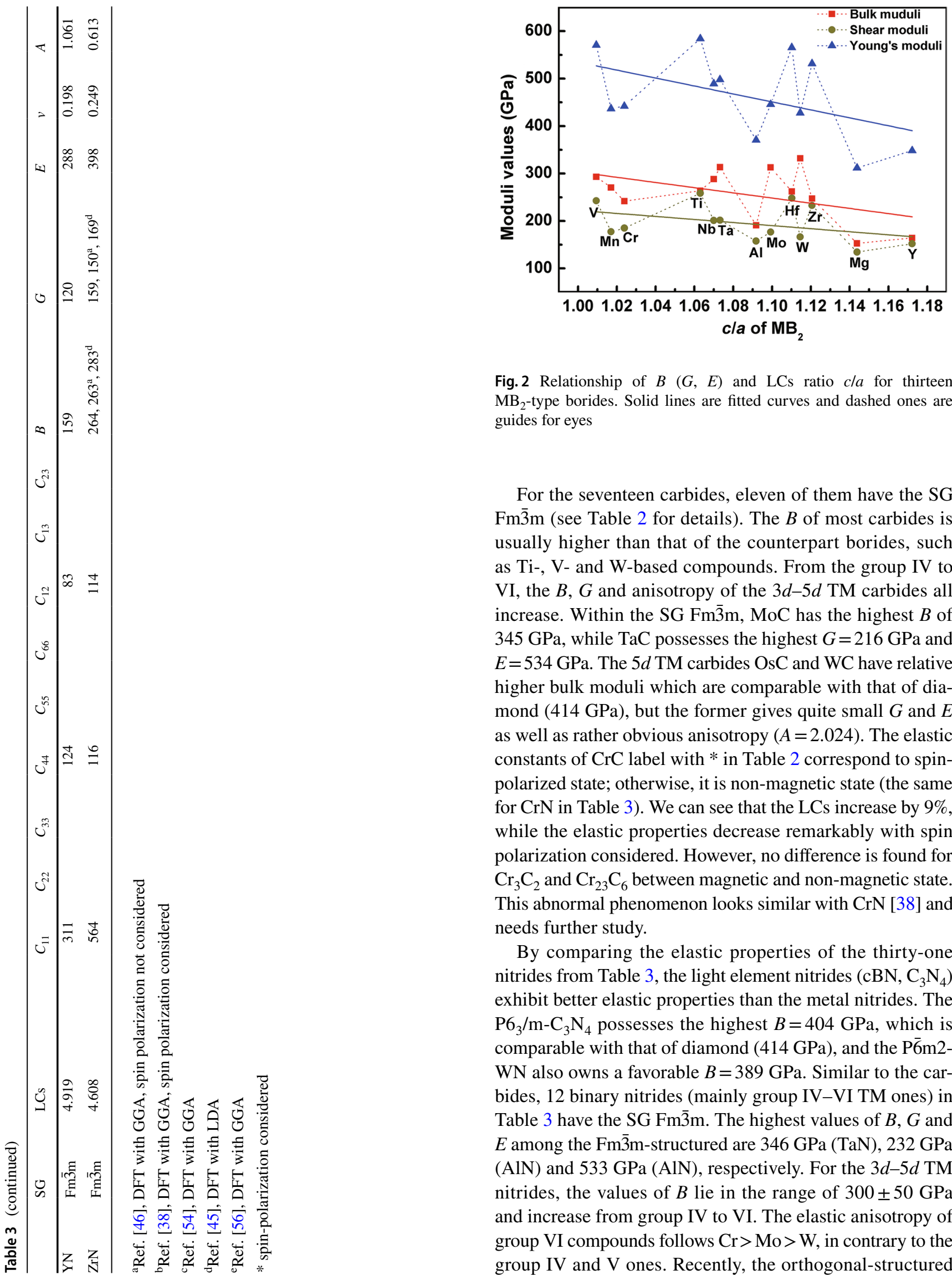

Fig. 2 Relationship of $B(G, E)$ and LCs ratio $c / a$ for thirteen $\mathrm{MB}_{2}$-type borides. Solid lines are fitted curves and dashed ones are guides for eyes

For the seventeen carbides, eleven of them have the SG Fm $\overline{3} \mathrm{~m}$ (see Table 2 for details). The $B$ of most carbides is usually higher than that of the counterpart borides, such as Ti-, V- and W-based compounds. From the group IV to VI, the $B, G$ and anisotropy of the $3 d-5 d$ TM carbides all increase. Within the SG Fm $\overline{3} \mathrm{~m}, \mathrm{MoC}$ has the highest $B$ of $345 \mathrm{GPa}$, while $\mathrm{TaC}$ possesses the highest $G=216 \mathrm{GPa}$ and $E=534 \mathrm{GPa}$. The $5 d \mathrm{TM}$ carbides OsC and WC have relative higher bulk moduli which are comparable with that of diamond (414 GPa), but the former gives quite small $G$ and $E$ as well as rather obvious anisotropy $(A=2.024)$. The elastic constants of $\mathrm{CrC}$ label with * in Table 2 correspond to spinpolarized state; otherwise, it is non-magnetic state (the same for $\mathrm{CrN}$ in Table 3). We can see that the LCs increase by $9 \%$, while the elastic properties decrease remarkably with spin polarization considered. However, no difference is found for $\mathrm{Cr}_{3} \mathrm{C}_{2}$ and $\mathrm{Cr}_{23} \mathrm{C}_{6}$ between magnetic and non-magnetic state. This abnormal phenomenon looks similar with $\mathrm{CrN} \mathrm{[38]} \mathrm{and}$ needs further study.

By comparing the elastic properties of the thirty-one nitrides from Table 3, the light element nitrides $\left(\mathrm{cBN}, \mathrm{C}_{3} \mathrm{~N}_{4}\right)$ exhibit better elastic properties than the metal nitrides. The $\mathrm{P}_{3} / \mathrm{m}-\mathrm{C}_{3} \mathrm{~N}_{4}$ possesses the highest $B=404 \mathrm{GPa}$, which is comparable with that of diamond (414 GPa), and the P $\overline{6} \mathrm{~m} 2$ WN also owns a favorable $B=389 \mathrm{GPa}$. Similar to the carbides, 12 binary nitrides (mainly group IV-VI TM ones) in Table 3 have the SG Fm $\overline{3} \mathrm{~m}$. The highest values of $B, G$ and $E$ among the $\mathrm{Fm} \overline{3} \mathrm{~m}$-structured are $346 \mathrm{GPa}(\mathrm{TaN}), 232 \mathrm{GPa}$ $(\mathrm{AlN})$ and $533 \mathrm{GPa}(\mathrm{AlN})$, respectively. For the $3 d-5 d \mathrm{TM}$ nitrides, the values of $B$ lie in the range of $300 \pm 50 \mathrm{GPa}$ and increase from group IV to VI. The elastic anisotropy of group VI compounds follows $\mathrm{Cr}>\mathrm{Mo}>\mathrm{W}$, in contrary to the group IV and V ones. Recently, the orthogonal-structured 


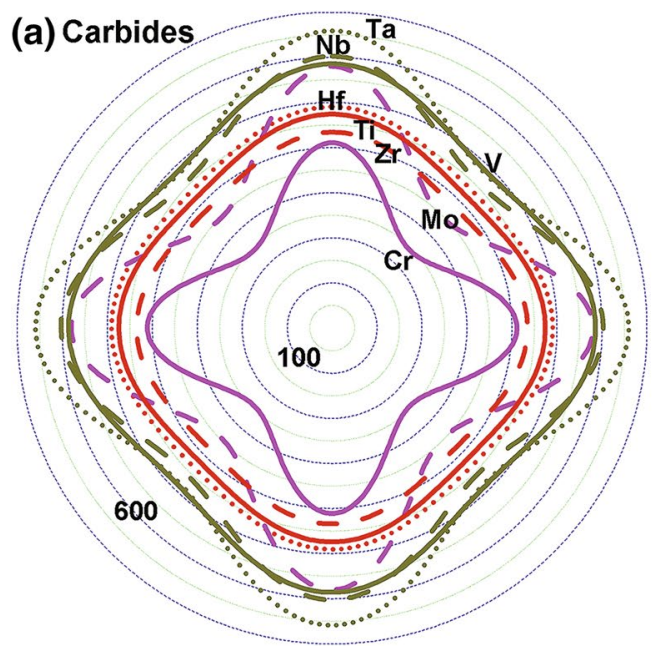

Fig. 3 Cross sections of Young's moduli distribution on the (001) plane ( $a b$ plane) for group IV-VI TM carbides a and nitrides $\mathbf{b}$. WC and $\mathrm{WN}$ are excluded for hexagonal structure, and $\mathrm{MoN}$ for elastic instability. Horizontal and vertical directions correspond to $a$ and $b$

$4 d$ and $5 d$ noble TMs Ir, Os, Pt and Ru nitrides have drawn much attention for their mechanical or functional properties $[42,49,56]$. Our calculations show their elastic properties are competitive with the group IV-VI TM nitrides; see details from Table 3. Again, clear difference is found for the LCs and elastic constants of $\mathrm{CrN}$ between magnetic and non-magnetic state. Zhou el al. [38] have discussed the influence of magnetic state on the elastic constants of $\mathrm{CrN}$, and the spin polarization should be employed in first-principles calculations.

Based on the above-calculated results of the ninety compounds, the $5 d$ TM phases $\mathrm{ReB}_{2}, \mathrm{WB}, \mathrm{OsC}, \mathrm{RuC}, \mathrm{WC}, \mathrm{OsN}_{2}$ and $\mathrm{WN}$ as well as TaN exhibit comparable bulk moduli

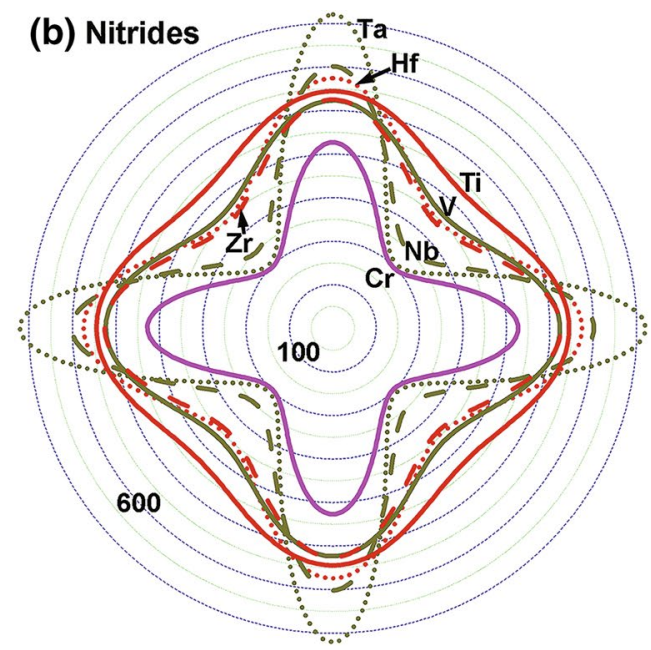

axes of a cubic crystal. Blue circles are contour lines and numbers are values with unit of GPa for Young's moduli; the increment is 100 $\mathrm{GPa}$

with $\mathrm{cBN}$; the others all have smaller bulk moduli. Therefore, one efficient way for screening hard materials is the synthesis of new compounds via strong covalent-bonded light elements or $5 d$ TMs with light elements. In addition, the fundamental elastic constants of these compounds can be further used to derive other elastic-related properties, such as Debye temperature [47].

\subsection{Anisotropy and Bonding}

The widely used group IV-VI TM compounds are representative among the ninety phases for their uniform cubic structure and considerable elastic properties. The elastic
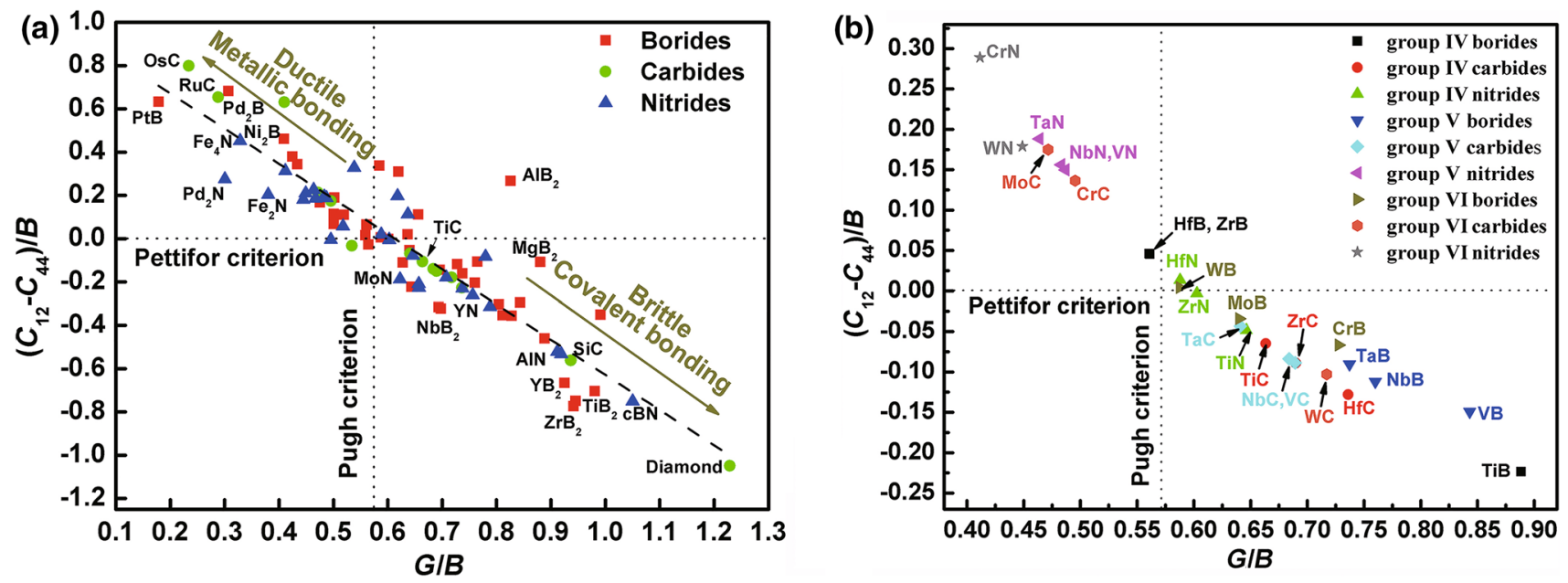

Fig. 4 Correlation between the normalized Cauchy pressures $p=\left(C_{12}-C_{44}\right) / B$ and the Pugh's modulus ratio $r=G / B$ for 86 elastically stable compounds $\mathbf{a}$ and group IV-VI TM phases $\mathbf{b}$. Black dash line in $\mathbf{a}$ is the fitting curve 
Table 4 Calculated SFEs at $0 \mathrm{~K}$. The SFEs of SF1 are italic

\begin{tabular}{|c|c|c|c|}
\hline & SFEs $\left(\mathrm{mJ} / \mathrm{m}^{2}\right)$ & & SFEs in \\
\hline & Cell shape fixed & Cell shape relaxed & \\
\hline Diamond & $277.5,9167$ & 258.1 & $318^{\mathrm{a}}, 279^{\mathrm{b}}$ \\
\hline $\mathrm{cBN}$ & $183.8,6515$ & 176.5 & $191^{\mathrm{c}}$ \\
\hline$\beta-\mathrm{SiC}$ & 1.52 & 1.14 & $-1.7^{\mathrm{d}}$ \\
\hline $\mathrm{PtN}$ & 318.0 & 293.7 & \\
\hline HfB & -26.2 & -46.6 & \\
\hline $\mathrm{ZrB}$ & 42.4 & 25.8 & \\
\hline $\mathrm{CrC}$ & $-100.7^{\mathrm{e}},-172.5^{\mathrm{f}}$ & $-198.7^{\mathrm{e}},-258.8^{\mathrm{f}}$ & \\
\hline $\mathrm{HfC}$ & 1609 & 1604 & \\
\hline $\mathrm{MoC}$ & -929.9 & -1211 & \\
\hline $\mathrm{NbC}$ & 637.8 & 568.5 & \\
\hline $\mathrm{ReC}$ & -1021 & -3206 & \\
\hline $\mathrm{TaC}$ & 772.6 & 633.4 & \\
\hline $\mathrm{TiC}$ & 1672,3495 & - & \\
\hline $\mathrm{VC}$ & 895.5 & 892.6 & \\
\hline $\mathrm{ZrC}$ & 1367 & - & \\
\hline AlN & 4813 & 4453 & \\
\hline $\mathrm{CrN}$ & $1112^{\mathrm{e}}, 2775^{\mathrm{f}}$ & $425.4^{\mathrm{e}}, 527.3^{\mathrm{f}}$ & \\
\hline $\mathrm{GaN}$ & 3544 & - & \\
\hline $\mathrm{HfN}$ & 234.5 & 297.9 & \\
\hline $\mathrm{InN}$ & 1821 & - & \\
\hline $\mathrm{NbN}$ & -130.0 & -677.5 & \\
\hline $\mathrm{ScN}$ & 1889 & - & \\
\hline $\mathrm{TaN}$ & -147.7 & -970.8 & \\
\hline $\mathrm{TiN}$ & 1081,1509 & - & $1050^{\mathrm{g}}$ \\
\hline $\mathrm{VN}$ & 91.0 & -329.1 & \\
\hline YN & 1217 & - & \\
\hline $\mathrm{ZrN}$ & 330.3 & 224.2 & \\
\hline
\end{tabular}

${ }^{\mathrm{a}}$ Ref. [62], DFT

${ }^{\mathrm{b}}$ Ref. [63], experimental result by transmission electron microscope

${ }^{\mathrm{c}}$ Ref. [64], experimental result by transmission electron microscope

${ }^{\mathrm{d}}$ Ref. [65], DFT

${ }^{\mathrm{e}}$ Spin polarization considered in this study

${ }^{\mathrm{f}}$ Spin polarization not considered in this study

${ }^{g}$ Ref. [66], DFT

anisotropy could be visualized by plotting Young's moduli $(E)$ versus crystal direction in cubic structure $[46,57]$. The larger the difference between the maximum and minimum values of the $E$, the stronger the anisotropy of the structure. The cross sections (perpendicular to $c$ axis of the crystal) of $E$ surface for the group IV-VI TM carbides and nitrides are shown in Fig. 3, where horizontal and vertical directions correspond to $a$ and $b$ axes of cubic crystals. For both carbides and nitrides, along the $a$ or $b$ direction, the $E$ is maximum, while minimum is along the bisector direction of $a$ and $b$ axes. From Fig. 3, the anisotropic character increases from group IV to group VI TM compounds. The group IV
TM (Ti, Zr and Hf) carbides are approximately isotropic, while group VI ( $\mathrm{Cr}$ and $\mathrm{Mo}$ ) carbides and nitrides show obvious elastic anisotropy. It should be also noted that these TM nitrides have stronger anisotropy than the counterpart carbides, especially for $\mathrm{HfN}, \mathrm{NbN}$ and TaN. These anisotropy characters are in good agreement with the factors calculated from Eq. (8) (see $A$ values in Tables 2, 3). Accordingly, discrepancies should be carefully considered in comparison between experimental and theoretical elastic properties for materials with strong anisotropy.

Based on the above-calculated elastic properties, the bonding characters can be analyzed by the normalized Cauchy pressures $p=\left(C_{12}-C_{44}\right) / B$ and the Pugh's modulus ratios $r=G / B$. If $p$ is negative and $r$ is higher than 0.57 , the material will appear to have covalent bonding; otherwise, it is metallic bonding [58-60]. Also, the lower the $p$ value, the higher the $r$ value and the more brittle the material. The relationship between $p$ and $r$ is plotted for the 86 elastically stable compounds (Fig. 4a) and group IV-VI TM phases (Fig. 4b). From Fig. 4a, most of these phases are distributed following the fitted curve (black dash line) $p=J * r+K$, where constants $J=-1.62 \pm 0.08$ and $K=1.00 \pm 0.05$. For borides and carbides, the group III-VI phases usually show covalent bonding and brittle characters, while group VII-X ones exhibit metallic bonding and ductile characters. For TM nitrides, the group III-IV phases have covalent bonding, while the others have metallic bonding. Although HfB and $\mathrm{ZrB}$ lie in the upper left region of Fig. 4b, all the group IV-VI TM borides can be regarded as covalent bonded. However, metallic bonding appears in the group V TM carbides as well as group VI TM carbides (besides WC) and nitrides. The origin of these bonding characters can be understood from electronic structure, which will be explored in our future work.

\subsection{Stacking Fault Energies}

Recent studies have confirmed that the Vickers hardness can increase from 90 to $120 \mathrm{GPa}$ and even $200 \mathrm{GPa}$ with single-crystalline, nano-grained and nano-twinned diamond [23]. The same phenomenon is also reported for $\mathrm{cBN}$ [22] and $\mathrm{Fm} \overline{3} \mathrm{~m}-\mathrm{SiC}(\beta-\mathrm{SiC})$ [24]. That is to say, these materials can be strengthened via grain refinement or twin defects. From this point of view, better elastic properties could be expected by introducing twins in the binary borides, carbides or nitrides. Twin formation tendency is strongly correlated with the SFEs, $\gamma$. It is easier for twin growth with lower SFE, such as $\beta-\operatorname{SiC}\left(\gamma \sim 1 \mathrm{~mJ} / \mathrm{m}^{2}\right)$ and silver $\left(\gamma \sim 18 \mathrm{~mJ} / \mathrm{m}^{2}\right)$; and it is rather difficult for twin formation in materials with high SFEs, such as aluminum $\left(\gamma \sim 130 \mathrm{~mJ} / \mathrm{m}^{2}\right)$ and diamond $\left(\gamma=277 \mathrm{~mJ} / \mathrm{m}^{2}\right)$. However, twins may form in high-SFE materials at certain conditions, e.g., high strain rate, high temperature and high pressure $[23,61]$. 
Considering the twined diamond, $\mathrm{cBN}$ and $\beta$-SiC are face-centered cubic (FCC) crystals, and thus the SFEs of twenty-seven compounds with FCC structure (SG F $\overline{4} 3 \mathrm{~m}$ for diamond, $\mathrm{cBN}, \beta-\mathrm{SiC}, \mathrm{PtN}$ and $\mathrm{Fm} \overline{3} \mathrm{~m}$ for the others) are calculated and listed in Table 4. Here, two regimes of relaxation are employed in the SFEs calculation, cell shape fixed and cell shape relaxed with the atom positions relaxed in both regimes. The SFEs of SF1 and SF2 configurations (illustrated in Fig. 1) have been tested for diamond, cBN, TiC and TiN. The energies of SF1 are italic in Table 4. We can see that the SFE2 is much lower than the SFE1 and indicates that SF2 is an energetically favorable configuration. Thus, only SF2 is considered for the other phases. With cell shape fixed regime, the calculated SFEs are in good agreement with the available data and slightly larger than the cell shape relaxed ones. Once the SFE is larger than $1000 \mathrm{~mJ} / \mathrm{m}^{2}$, only the fixed regime is employed because there is little difference between the two regimes, e.g., HfC and AlN; see Table 4 for details. Nano-twinned diamond [23] can be fabricated from onion-like precursor. Thus, at certain circumstance, twins may form in $\mathrm{PtN}, \mathrm{ZrB}, \mathrm{HfN}, \mathrm{VN}$ and $\mathrm{ZrN}$, whose SFEs are lower or slightly higher than that of diamond $(\gamma=277.5 \mathrm{~mJ} /$ $\mathrm{m}^{2}$ ) from Table 4 . Thus, the mechanical performance could be enhanced via twinning. However, it is difficult for twin formation in most of the carbides and nitrides, which have rather large SFEs, i.e., $\gamma>\gamma(\mathrm{NbC})=637.8 \mathrm{~mJ} / \mathrm{m}^{2}$. The reason why several SFEs ( $\mathrm{HfB}, \mathrm{CrC}, \mathrm{MoC}, \mathrm{ReC}, \mathrm{NbN}$ and $\mathrm{TaN}$ ) are much below zero is that the energy of the faulted stacking sequence is lower than perfect FCC stacking configuration. In other words, a hexagonal structure, in which close-packed stacking sequence is the same as the faulted FCC stacking sequence, is more energetically stable than the FCC structure with the same atoms. For instance, the energy of one Ta atom plus one $\mathrm{N}$ atom in $\mathrm{P}_{3} / \mathrm{mmc}-\mathrm{TaN}$ is $-22.112 \mathrm{eV}$, which is lower than $-21.924 \mathrm{eV}$ for $\mathrm{Fm} \overline{3} \mathrm{~m}-\mathrm{TaN}$ and thus results in negative SFE.

\section{Conclusion}

Enormous first-principles calculations are employed in the determination of elastic properties and SFEs of ninety binary borides, carbides and nitrides, and the calculations show reasonable agreement with the available data. Most of these compounds have a considerable bulk modulus more than 260 $\mathrm{GPa}$, especially for the $5 d \mathrm{TM}$ ones $\left(\mathrm{ReB}_{2}, \mathrm{WB}, \mathrm{OsC}, \mathrm{RuC}\right.$, $\mathrm{WC}, \mathrm{OsN}_{2}$, TaN and $\mathrm{WN}$ ), in which the bulk moduli are comparable with cBN. Light element phases exhibit superior elastic properties than TM ones. Therefore, the synthesis of new compounds via strong covalent-bonded light elements or $5 d$ TMs with light elements is an efficient way to be harder. The group IV-VI TM borides show covalent bonding, while metallic bonding appears in the group VI TM carbides, the group VI TM nitrides and the group V carbides. The anisotropy characters are distinguishing for the group IV-VI TM carbides and nitrides. Another way for getting harder is introducing twin boundary defects into the materials. By comparing the calculated SFEs of twentyseven cubic compounds, twins can be expected to form in $\mathrm{ZrB}$, HfN, PtN, VN and $\mathrm{ZrN}$, since their SFEs are lower or slightly higher than that of diamond.

Acknowledgements This work was supported by the National Natural Science Foundation of China (Nos. 11427806, 51471067, 51671082, 51671086 and 51302313) and the National Key Research and Development Program of China (No. 2016YFB0300801). We are highly grateful for the kind help from Zhixiao Liu at Hunan University, Changsha, with the written English.

\section{References}

[1] H.O. Pierson, Handbook of Refractory Carbides and Nitrides (Noyes Publications, Westwood, 1996)

[2] R.B. Kaner, J.J. Gilman, S.H. Tolbert, Science 308, 1268 (2005)

[3] A.L. Ivanovskii, Prog. Mater. Sci. 57, 184 (2012)

[4] R. Telle, L.S. Sigl, K. Takagi, Boride-based hard materials, in Handbook of Ceramic Hard Materials (Wiley-VCH Verlag GmbH, Weinheim, 2008), pp. 802-945

[5] P.H. Mayrhofer, C. Mitterer, L. Hultman, H. Clemens, Prog. Mater. Sci. 51, 1032 (2006)

[6] G.F. Huang, L.P. Zhou, W.Q. Huang, L.H. Zhao, S.L. Li, D.Y. Li, Diam. Relat. Mater. 12, 1406 (2003)

[7] J. Xiang, Z. Xie, Y. Huang, H. Xiao, J. Eur. Ceram. Soc. 20, 933 (2000)

[8] K.B. Panda, K.S. Ravi Chandran, Metall. Mater. Trans. A 34, 1371 (2003)

[9] Y. Katoh, K. Ozawa, C. Shih, T. Nozawa, R.J. Shinavski, A. Hasegawa, L.L. Snead, J. Nucl. Mater. 448, 448 (2014)

[10] A. Iveković, S. Novak, G. Dražić, D. Blagoeva, S.G. de Vicente, J. Eur. Ceram. Soc. 33, 1577 (2013)

[11] J. Hu, H. Xiao, W. Guo, Q. Li, W. Xie, B. Zhu, Ceram. Int. 40, 1065 (2014)

[12] J. Nagamatsu, N. Nakagawa, T. Muranaka, Y. Zenitani, J. Akimitsu, Nature 410, 63 (2001)

[13] H. Dong, H.X. Yang, X.P. Ai, C. Cha, Int. J. Hydrog. Energy 28, 1095 (2003)

[14] A. Zerr, G. Miehe, G. Serghiou, M. Schwarz, E. Kroke, R. Riedel, H. Fuesz, P. Kroll, R. Boehler, Nature 400, 340 (1999)

[15] E. Gregoryanz, C. Sanloup, M. Somayazulu, J. Badro, G. Fiquet, H.K. Mao, R.J. Hemley, Nat. Mater. 3, 294 (2004)

[16] J.C. Crowhurst, A.F. Goncharov, B. Sadigh, C.L. Evans, P.G. Morrall, J.L. Ferreira, A.J. Nelson, Science 311, 1275 (2006)

[17] R.S. Igor, L.I. Alexander, J. Phys. Condens. Matter 20, 415218 (2008)

[18] Z.S. Zhao, L.F. Xu, L.M. Wang, B. Xu, M. Wang, Z.Y. Liu, J.L. He, Comput. Mater. Sci. 50, 1592 (2011)

[19] Y.H. Duan, Y. Sun, Z.Z. Guo, M.J. Peng, P.X. Zhu, J.H. He, Comput. Mater. Sci. 51, 112 (2012)

[20] X.F. Hao, Y.H. Xu, Z.J. Wu, D.F. Zhou, X.J. Liu, X.Q. Cao, J. Meng, Phys. Rev. B 74, 224112 (2006)

[21] V.V. Brazhkin, A.G. Lyapin, R.J. Hemley, Philos. Mag. A 82, 231 (2002) 
[22] Y.J. Tian, B. Xu, D.L. Yu, Y.M. Ma, Y.B. Wang, Y.B. Jiang, W.T. Hu, C.C. Tang, Y.F. Gao, K. Luo, Z.S. Zhao, L.M. Wang, B. Wen, J.L. He, Z.Y. Liu, Nature 493, 385 (2013)

[23] Q. Huang, D.L. Yu, B. Xu, W.T. Hu, Y.M. Ma, Y.B. Wang, Z.S. Zhao, B. Wen, J.L. He, Z.Y. Liu, Y.J. Tian, Nature 510, 250 (2014)

[24] Z.J. Lin, L. Wang, J.Z. Zhang, X.Y. Guo, W. Yang, H.K. Mao, Y.S. Zhao, Scr. Mater. 63, 981 (2010)

[25] T.F. Li, T.M. Liu, L.Q. Zhang, T. Fu, H.M. Wei, Comput. Mater. Sci. 126, 103 (2017)

[26] B. Yang, X. Peng, H. Xiang, D. Yin, C. Huang, S. Sun, T. Fu, J. Alloys Compd. 739, 431 (2018)

[27] E.O. Hall, Proc. Phys. Soc. Lond. Sect. B 64, 747 (1951)

[28] N.J. Petch, J. Iron Steel Inst. 174, 25 (1953)

[29] Y. Zhang, N.R. Tao, K. Lu, Scr. Mater. 60, 211 (2009)

[30] X.H. An, Q.Y. Lin, S.D. Wu, Z.F. Zhang, R.B. Figueiredo, N. Gao, T.G. Langdon, Scr. Mater. 64, 954 (2011)

[31] D.T. Pierce, J.A. Jiménez, J. Bentley, D. Raabe, C. Oskay, J.E. Wittig, Acta Mater. 68, 238 (2014)

[32] S. Kibey, J.B. Liu, D.D. Johnson, H. Sehitoglu, Acta Mater. 55, 6843 (2007)

[33] G. Kresse, J. Furthmüller, Phys. Rev. B 54, 11169 (1996)

[34] G. Kresse, J. Furthmüller, Comput. Mater. Sci. 6, 15 (1996)

[35] G. Kresse, D. Joubert, Phys. Rev. B 59, 1758 (1999)

[36] J.P. Perdew, K. Burke, M. Ernzerhof, Phys. Rev. Lett. 77, 3865 (1996)

[37] H.J. Monkhorst, J.D. Pack, Phys. Rev. B 13, 5188 (1976)

[38] L. Zhou, F. Körmann, D. Holec, M. Bartosik, B. Grabowski, J. Neugebauer, P.H. Mayrhofer, Phys. Rev. B 90, 184102 (2014)

[39] R. Yu, J. Zhu, H.Q. Ye, Comput. Phys. Commun. 181, 671 (2010)

[40] R. Hill, Proc. Phys. Soc. Lond. Sect. A 65, 349 (1952)

[41] K. Lau, A.K. McCurdy, Phys. Rev. B 58, 8980 (1998)

[42] Z.J. Wu, E.J. Zhao, H.P. Xiang, X.F. Hao, X.J. Liu, J. Meng, Phys. Rev. B 76, 054115 (2007)

[43] L.H. Liu, J.H. Chen, T.W. Fan, Z.R. Liu, Y. Zhang, D.W. Yuan, Comput. Mater. Sci. 108, 136 (2015)

[44] Q.Q. Shao, L.H. Liu, T.W. Fan, D.W. Yuan, J.H. Chen, J. Alloys Compd. 726, 601 (2017)
[45] Z.G. Wu, X.J. Chen, V.V. Struzhkin, R.E. Cohen, Phys. Rev. B 71, $214103(2005)$

[46] M.G. Brik, C.G. Ma, Comput. Mater. Sci. 51, 380 (2012)

[47] G.V. Sin'ko, N.A. Smirnov, J. Phys. Condens. Matter 14, 6989 (2002)

[48] K.B. Panda, K.S.R. Chandran, Acta Mater. 54, 1641 (2006)

[49] S.K.R. Patil, S.V. Khare, B.R. Tuttle, J.K. Bording, S. Kodambaka, Phys. Rev. B 73, 104118 (2006)

[50] T. Oguchi, J. Phys. Soc. Jpn. 71, 1495 (2002)

[51] J.Q. Qin, D.W. He, J.H. Wang, L.M. Fang, L. Lei, Y.J. Li, J. Hu, Z.L. Kou, Y. Bi, Adv. Mater. 20, 4780 (2008)

[52] Y.F. Li, Y.M. Gao, B. Xiao, T. Min, Y. Yang, S.Q. Ma, D.W. Yi, J. Alloys Compd. 509, 5242 (2011)

[53] C. Jiang, Appl. Phys. Lett. 92, 041909 (2008)

[54] X.G. Lu, M. Selleby, B. Sundman, Acta Mater. 55, 1215 (2007)

[55] Y. Liang, J. Zhao, B. Zhang, Solid State Commun. 146, 450 (2008)

[56] W. Chen, J.S. Tse, J.Z. Jiang, Solid State Commun. 150, 181 (2010)

[57] A. Cazzani, M. Rovati, Int. J. Solids Struct. 40, 1713 (2003)

[58] S.F. Pugh, Philos. Mag. 45, 823 (1954)

[59] D.G. Pettifor, Mater. Sci. Technol. 8, 345 (1992)

[60] Y. Zhang, D.W. Yuan, J.H. Chen, G. Zeng, T.W. Fan, Z.R. Liu, C.L. Wu, L.H. Liu, J. Electron. Mater. 45, 4018 (2016)

[61] F. Zhao, L. Wang, D. Fan, B.X. Bie, X.M. Zhou, T. Suo, Y.L. Li, M.W. Chen, C.L. Liu, M.L. Qi, M.H. Zhu, S.N. Luo, Phys. Rev. Lett. 116, 075501 (2016)

[62] P. Käckell, J. Furthmüller, F. Bechstedt, Phys. Rev. B 58, 1326 (1998)

[63] P. Pirouz, D.J.H. Cockayne, N. Sumida, P. Hirsch, A.R. Lang, Proc. R. Soc. Lond. Ser. A 386, 241 (1983)

[64] L. Nistor, S. Nistor, G. Dinca, J. Van Landuyt, D. Schoemaker, V. Copaciu, P. Georgeoni, N. Arnici, Diam. Relat. Mater. 8, 738 (1999)

[65] U. Lindefelt, H. Iwata, S. Öberg, P.R. Briddon, Phys. Rev. B 67, 155204 (2003)

[66] S.K. Yadav, X.Y. Liu, J. Wang, R. Ramprasad, A. Misra, R.G. Hoagland, Philos. Mag. 94, 464 (2014) 